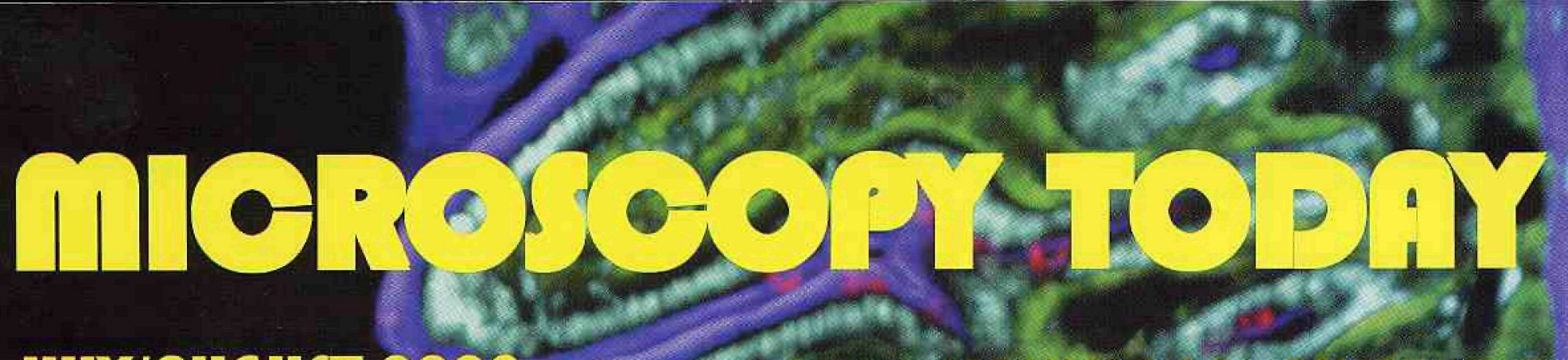
JUTY/QUGUST 2002

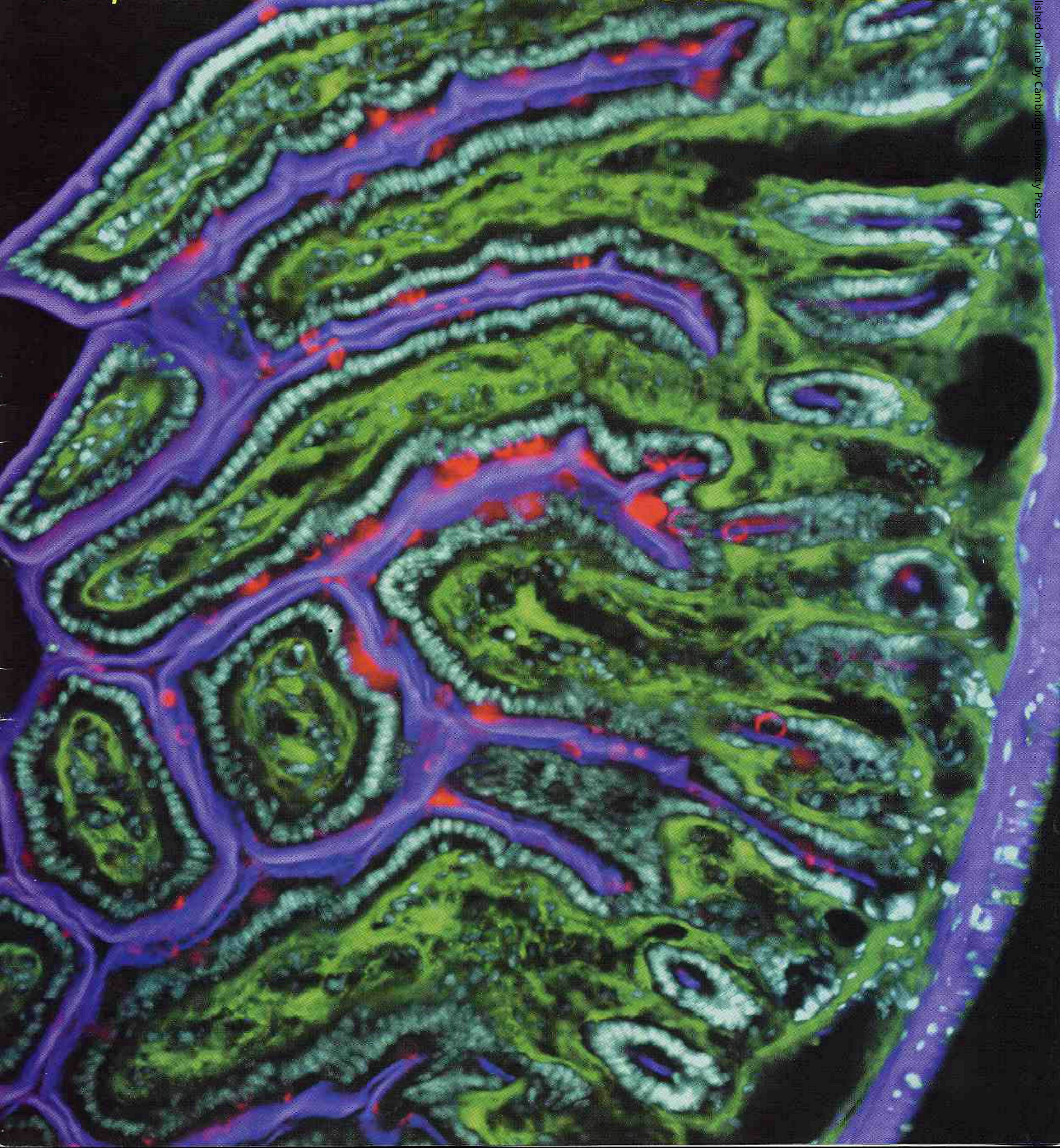




\section{very tiny bar codes}

Stephen W. Carmichael, ${ }_{2}^{1}$ Mayo Clinic

carmichael.stephen@mayo.edu

The use of barcodes on products, combined with the computer technology to read and track the subsequent data, has revolutionized inventory control in our society. Barcodes are commonly seen on items as large as automobiles and as smal as pieces of candy. But if you need to track a large number of items that are microscopic in size, such as biologically relevant molecules (think genomics, proteomics, metabolomics, etc.), then you need very small barcodes. Sufficiently small rods that can be marked as barcodes have been developed by Sheila Nicewarner-Peña, Griffith Freeman, Brian Reiss, Lin He, David Peña, Ian Walton, Remy Cromer, Christine Keating, and Michael Natan. ${ }^{2}$

Their starting point was a membrane of alumina with pores that served as a mold for the rods. The backside of the membrane was coated with a silver film that served as the working electrode. Using apparently simple electrochemistry, different metals were sequentially deposited within the pores, creating metallic stripes along the rod. The rod structure was determined by (i) the membrane pore diameter, that determines the diameter of the rod, (ii) the sequence of metal ions introduced into the solution bathing the membrane, that defines the number and pattern of metal stripes, and (iii) the electrical charge passed in each step, that dictates the length of each stripe. Nicewarner-Peña et al. have used seven different metals (cobalt, copper, gold, nickel, palladium, platinum, and silver) to create stripes as short as 10 nanometers and as long as several micrometers, and have prepared rods with as many as 13 distinguishable stripes.

After the metals are deposited within the pores, the membrane is dissolved with nitric acid and sodium hydroxide isolating the rods. The rods were examined by transmission electron microscopy, scanning electron microscopy (SEM), field emission SEM, energy-dispersive x-ray scattering, macroscopic extinction, and microscopic reflectivity, in addition to chemical analyses typically applied to bulk materials. Most significantly, examination with the light microscope ${ }^{3}$ clearly revealed a pattern of stripes when examined at the appropriate wavelength. Various metals have different reflectivities at different wavelength. For example, it was determined that at a wavelength of 430 nanometers, silver was 2.5 times more reflective than gold, whereas other wavelengths worked better for other combinations of metals. This suggests that reading the stripes may require more than one wavelength to optimize the number of unique barcodes.

The number of unique barcode patterns of $n$ number of metals with s number of stripes is on the order of $n^{3}$. Although the limit of resolution in a typical light microscope is around 143 nanometers, Nicewarner-Peña et al. suggested stripes on the order of half a micron. With a rod a few microns in length, this gives tens of thousands of unique combinations, far more than any other tagging method yet developed.

Fortunately, there are many chemical methods for attaching biologic molecules to the barcoded rods. Nicewarner-Peña et al. demonstrated this by attaching DNA molecules to rods that were then used in hybridization assays, and immunoglobulins used in a sandwich immunoassay. As the field of bioinformatics explodes and interest grows in conducting comprehensive analyses on classes of molecules, there will be a demand for very high-level multiplexing in small samples. This in turn will depend on a suitably small readout system. The barcodes developed by Nicewarner-Peña et al. may have fulfilled this need of the future.

\section{References}

1 The author gratefully acknowledges Dr. Christine D. Keating for reviewing this article.

2 Nicewarner-Peña, S.R., R.G. Freeman, B.D. Reiss, L. He, D.J. Peña, I.D. Walton, A. Cromer, C.D. Keating, and M.J. Natan, Submicrometer metallic barcodes, Science 294:137-141, 2001.

3 They used a Nikon TE-300 inverted microscope equipped with a brightfield reflectance filter, using $100 \mathrm{X}$ oil immersion lens, and a bandpass filter to vary the wavelength of the excitation beam.

\section{InDEX Or Anutotes}

very tiny bar codes

Stephen W. Carmichael, Mayo Clinic

Prog. Towards More Realistic In-Situ Microscopy Observations ...... 5

A. Howie, Cavendish Laboratory, University of Cambridge

Spectral Image Analysis: Getting The Most From All That Data ....... 8

P.G. Kotula, M.R. Keenan, and R. Loehman, Sandia National Laboratories New Fluorescent Labeling Technologies for Ultrasensitive Cytochemical and Histochemical Imaging

Iain Johnson, Molecular Probes, Inc

Artifacts \& Non-Local Effects In SPM Potential Measurements 16

Sergei V. Kalinin and Dawn A. Bonnell, University of Pennsylvania

Vibration, Resonance and the Effect on Microscopes

Douglas A. Anderson, Schnabel Engineering Associates

The Basics of Immunoglobulins and Immunostaining

W. Gray (Jay) Jerome, Vanderbilt University Medical Center

Swapping Atoms For Bits: Managing The Digital Evolution In The

Microscopy Laboratory

Judy A. Murphy, San Joaquin Delta College

A Note on lodine and Vacuum Pumps

Scott D. Walck, PPG Industries, Inc.

A Simple Way to Eliminate Frost Build-up on Cryo-SEM Samples. 34

Gib Ahlstrand, University of Minnesota
3 Mousescope ${ }^{T M}$

Lee van Hook, Piltdown Research Institute, Münchhausen University

A Method for Safely Restraining Mouse Pups for Microscopy .........36

Michael J. Herron, University of Minnesota

A Simple Image Archive That's Cheap, Too!

Tim Morken, Centers for Disease Control and Prevention

\section{BOUT TIIE GOVER}

\section{lain Johnson, Molecular Probes, Inc. See his article on labelling technologies on page 12}

A cryosection of mouse intestine showing basement membranes labeled with an anti-fibronectin antibody and Alexa Fluor 488 goat anti-chicken secondary antibody (green fluorescence). Goblet cells and crypt cells are labeled with Alexa Fluor 594 wheat germ agglutinin (red fluorescence). The microvillar brush border and smooth muscle layer are visualized with Alexa Fluor 680 phalloidin 